\title{
Morphological Changes Induced by the Absence of Ovarian Hormones in Nucleus Accumbens of Ovariectomized Rats
}

\author{
Alma Rosa Quiñones ${ }^{1}$, Israel Camacho-Abrego ${ }^{2}$, Nancy Antonia Rivera ${ }^{2}$, Gonzalo Flores ${ }^{2}$ and \\ Ofir Picazo ${ }^{*}, 1$ \\ ${ }^{\text {I}}$ Escuela Superior de Medicina del I.P.N., Plan de San Luis y Diaz Mirón, Col. Sto. Tomás, México D.F. 11340, México \\ ${ }^{2}$ Laboratorio de Neuropsiquiatría, Instituto de Fisiología, Universidad Autónoma de Puebla. 14 Sur 6301, CP: 72570, \\ Puebla, México
}

\begin{abstract}
Ovariectomy (OVX) has been widely used to explore the effect of ovarian hormones on brain regions. It has been observed that estrogen in OVX rats increases the dopaminergic function in nucleus accumbens (NAcc). To determine whether absence or presence of estradiol changes the dendritic structure of some dopaminergic neurons, we investigated the consequences of a daily injection for 2 weeks of 17-beta estradiol (E2) on the dendritic morphology of NAcc neurons in two groups of rats, immediately or 10 weeks administered after OVX. The structure of dendrites was measured by using the Golgi-Cox procedure followed by a Sholl analysis. We found a reduction in the number of spiny dendrites of the medium spiny neurons of the NAcc in animals in which E2 was administrated without delay after OVX. However, when the same treatment was administered 10 weeks after OVX, not any difference was observed. Independently of the hormone treatment, rats from the group of 10 weeks after OVX showed a reduction in the number of spiny dendrites of the medium spiny neurons when compared with their counterparts at 2 weeks. Data suggest that estrogens or their absence regulate synaptogenesis in the NAcc.
\end{abstract}

\section{INTRODUCTION}

The decline of the ovarian function occurring in aged females has been related to many somatic and psychological disturbances [1-5], changes in several neurotransmitters and their receptors [6-9], and variations in the fine neuronal structures in several brain regions [10-12]. Thus, while the ovarian hormones estrogen and progesterone are able to modify the dendritic spine density and the morphology of neurons from various brain regions [13-15], their absence induced through an ovariectomy (OVX), can affect the dendritic arbor of such structures [12-14, 16, 17]. However, the influence of estrogens on dendritic structure at NAcc has not yet been documented.

Theoretically, the gradual reduction of steroid hormones characterizing the menopausal period can be mimicked by the removal of ovaries. This manipulation has been proposed as a model for studying post-menopausal osteoporosis in humans [18-20] and recently has also been used to show that some behavioral symptoms associated with human postmenopause can be studied in rats OVX 12 weeks previously [4]. The current study utilized this same model to explore possible changes in the neuronal morphology of NAcc, influenced by the absence of ovarian hormones. These results were compared to rats treated with E2 for two weeks, either immediately or ten weeks after OVX. The Golgi-Cox

*Address correspondence to this author at the Escuela Superior de Medicina del I.P.N., Plan de San Luis y Diaz Mirón, Col. Sto. Tomás, México D.F. 11340, México; Tel: (xx) 55 57296300, Ext. 62761; Fax, Ext. 62806;

E-mail: rifo99mx@yahoo.com.mx, rifo99@hotmail.com stain method and Sholl analysis were employed to determine dendritic length, neuronal branching and spine density of spiny medium neurons of the NAcc.

\section{MATERIALS AND METHODOLOGY}

Forty female Wistar rats were obtained from our animal facility (Escuela Superior de Medicina-IPN, ESM-IPN). All rats weighed between 225 and $250 \mathrm{~g}$ at approximately 3 months of age. Animals were housed in pairs and maintained in a temperature and humidity controlled environment, with free access to food and water. All surgical procedures described in this study were approved by the ESM-IPN Animal Care Committee and met governmental guidelines (Mexican Council for Animal Care, Norma Oficial Mexicana NOM-062-ZOO-1999). All efforts were made to minimize both animal suffering and to reduce the number of animals used. All animals included in this study were ovariectomized through a dorsal incision under 2,2,2, tribromoethanol anesthesia $(0.2 \mathrm{~g} / \mathrm{kg}$, i.p.). The complete extraction of the ovaries was corroborated by visual inspection. Post-surgical care included administration of the antibiotic Baytril $(0.03$ $\mathrm{ml} /$ animal, SC) and analgesic banamine $(0.03 \mathrm{ml} / \mathrm{animal}$, $\mathrm{SC})$. Subsequent to surgery, all animals were assigned to two groups, the first group received immediately a daily subcutaneous injection of corn oil $(1 \mathrm{ml} / \mathrm{kg}, \mathrm{n}=10)$ or $17-\beta$ estradiol benzoate $(50 \mu \mathrm{g} / \mathrm{kg} \mathrm{n}=10)$ during two weeks after OVX. The second group received the same treatment ten weeks after the surgery $[(10-w-O V X) \quad(n=10$, each for vehicle and E2)]. Doses and treatments were selected according to previous reports [21]. All injections were done between 800-1000 h each day. 
One day after the last E2 injection, rats were deeply anesthetized with sodium pentobarbital $(60 \mathrm{mg} / \mathrm{kg}$ body weight, ip) and perfused intracardially with $0.9 \%$ saline solution. The brains were rapidly removed and processed by the modified Golgi-Cox method [22]. Briefly, the brains were stored in the dark first in the Golgi-Cox solution for 14 days and then in a $30 \%$ sucrose solution for 3 days. The brains were then blocked and sliced coronally in $200 \mu \mathrm{m}$ thick sections at the level of the NAcc using a vibratome (Campden Instrument, MA752, Leicester, England). The sections were collected on clean, $2 \%$ gelatin-coated glass microscope slides ( 4 sections/slide). The mounted tissue was rinsed in distilled water and then placed in a bath of ammonium hydroxide for $30 \mathrm{~min}$ in the dark. After rinsing, the sections were then immersed in the dark in Kodak Film Fixer for $30 \mathrm{~min}$ and subsequently washed with distilled water, dehydrated and cleared in successive baths of $50 \%$ (1 $\mathrm{min}), 70 \%(1 \mathrm{~min}), 95 \%(1 \mathrm{~min})$ and $100 \%(2 \times 5 \mathrm{~min})$ alcohol followed by $15 \mathrm{~min}$ in a xylene solution. The slides were covered with balsam resinous medium [23, 24].

Medium spiny neurons of the NAcc (plate 9 - 12) of Paxinos and Watson Atlas [25] were selected for this study. For each animal, five neurons from both the left and right NAcc were drawn using camera lucida at a magnification of 250X (DMLS, Leica Microscope) by a trained observer, who was blind to the experimental conditions [26]. Medium spiny neurons from the NAcc were identified by soma size and dendritic extension, as described by Robinson and Kolb [27] and Flores et al., [22]. The criteria used to select neurons for reconstruction have been described in details elsewhere [22, 28-30]. Briefly, only complete, fully impregnated pyramidal neurons with no apparent truncation of basal dendritic arbor were included in our analyses; terminals were positively identified by their characteristic conical shape. Our analyses were performed on basal dendrites of pyramidal neurons as they are parallel to the coronal plane. A student blind to the experimental procedures was assigned to observe, identify and reconstruct the three-dimensional dendritic tree by drawing each neuron in a two dimensional plane. The dendritic tree was studied by Sholl analysis as follows: a transparent grid with concentric rings, equivalent to $10 \mu \mathrm{m}$ apart, was placed over the dendritic drawing and the number of ring intersections were used to estimate total dendritic length [23, 31]. Another estimate of dendritic arborization is the total number of dendritic branches (branching indicated by bifurcation) which were counted at each order away from the cell body or dendritic shaft. Finally, the density of dendritic spines was estimated by randomly selecting highmagnification $(1,000 \mathrm{X})$ tracings of a $10-\mu \mathrm{m}$-long terminal segment of the dendritic branch; in most cases the length of the selected distal branch exceeded $40 \mu \mathrm{m}$. Estimates of spine density were generated by counting the number of visible spines along the branch segment and expressing the data as number of spines $/ 10 \mu \mathrm{m}$.

Mean values from NAcc of each animal were treated as a single measurement for the data analysis. Data on dendritic length and spine densities were analyzed by two-way ANOVA, followed by Newman-Keuls test for post-hoc comparisons, with E2 treatment and time after OVX as independent factors. Data from length per branch order were analyzed using a two-way ANOVA, followed by Bonferroni test for post-hoc comparisons, with E2 administration and branch order as independent factors $(\mathrm{P}<0.05$ was considered significant).

\section{RESULTS}

The morphological analysis presented here is based on 400 NAcc medium spiny neurons from 40 animals and the E2 effects on dendritic morphology in the NAcc from ovariectomized animals are illustrated in Fig. (1). The neuronal dendritic morphology of NAcc neurons was measured by Golgi-Cox stain and dendritic length for each branching order, spine density and total dendritic length were obtained as reported previously [22, 24, 28-30]. The GolgiCox impregnation procedure clearly filled the dendritic shafts and the spines of neurons from Nacc (Fig. 1A).

The two-way ANOVA analysis revealed a significant effect of time after OVX $\left(\mathrm{F}_{1,35}=13, P<0.001\right)$ and interaction of E2 and time $\left(\mathrm{F}_{1,35}=6.1, P=0.01\right)$ in dendritic spine density of medium spiny neurons of the NAcc (Fig. 1B). Post-hoc test revealed that dendritic spine density was significantly lower in the medium spiny neurons of the NAcc from the immediatelyOVX rats with E2 administration compared to their corresponding vehicle group $(P<0.01)$. Interestingly, the E2 administration in the $10-\mathrm{w}-\mathrm{OVX}$ rats did not produce any difference (Fig. 1B). The analysis between vehicle groups revealed that dendritic spine density was significantly lower in the medium spiny neurons of the NAcc from 10 -w-OVX rats with vehicle compared to immediately-OVX rats with vehicle as well $(P<0.001)$ (Fig. 1B). The total dendritic length of medium spiny NAcc neurons (Two-way ANOVA, between E2: $\mathrm{F}_{1,35}=1.9, P=0.1$; time post-OVX: $\mathrm{F}_{1,35}=0.5, P=0.1$ and interaction E2 and time post-OVX $\mathrm{F}_{1,35}=0.45, P=0.5$ ) revealed no difference between groups (Fig. 1C). Two-way ANOVA for the analysis of length per branch order of medium spiny NAcc neurons from immediately-OVX rats with $\mathrm{E} 2$, revealed a significant effect of branch order $\left(\mathrm{F}_{1,35}=88, P<0.01\right)$, as well as an interaction of this factor with $\mathrm{E} 2$ administration $\left(\mathrm{F}_{1,35}=2.05\right.$, $P=0.04$ ) (Fig. 1D). Post-hoc test revealed that the dendritic length at the level of the third order decreased in the immediately-OVX rats with E2 administration compared to corresponding vehicle groups $(P<0.05)($ Fig. 1D). Finally, twoway ANOVA for the analysis of length per branch order of medium spiny NAcc neurons from 10-w-OVX rats with E2 did not show any differences (Fig. 1E).

\section{DISCUSSION}

These data evidence that E2 induces a differential effect, which is dependent on the time of hormone administration after OVX. Thus, a reduction in the number of spiny dendrites of the medium neurons of the NAcc in the animals immediately treated (for two weeks) with the hormone after OVX, compared to the respective control, was observed. In contrast, no difference was found with the same treatment administered 10 weeks after the surgery. In addition, rats from 10 -w-OVX control group showed a reduction in the number of spiny dendrites analyzed in comparison to immediately-OVX control group. Data suggest that estrogens or their absence regulate synaptogenesis in the NAcc.

The mechanism by which E2 induces changes in dendritic architecture at NAcc is not clear. However, both dendritic growth and spiny density on dendrites seem to be 

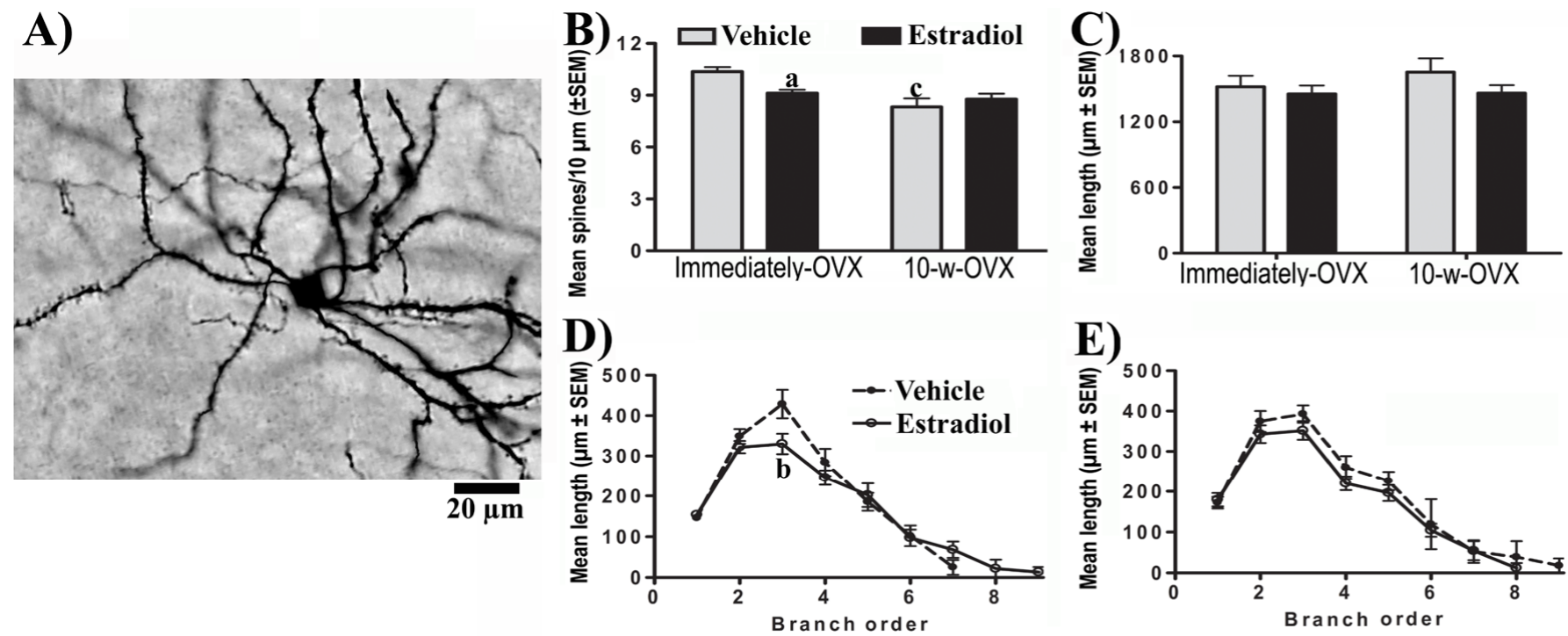

Fig. (1). Analysis of NAcc medium spine neurons in rats with estrogenic treatment for two weeks at two different post-OVX times, immediately after- and 10 weeks after-OVX $(n=10$ animals per group). (A) Golgi-Cox-impregnated nucleus accumbens neuron from a vehicle treated animal immediately after OVX. (B) Spine density decreased in immediately-OVX rats with E2 compared to their corresponding vehicle group $\left({ }^{\mathrm{a}} \mathrm{P}<0.01\right)$. The E2 administration in the 10 -w-OVX rats did not produce any difference. Interestingly, 10 -wOVX group with vehicle also showed significant decrease in spine density compared to immediately-OVX rats with vehicle $\left({ }^{\mathrm{c}} P<0.001\right)$. $(\mathbf{C})$ Total dendritic length analysis revealed no differences among groups. (D) Length of branch order of the rats treated with E2 immediately after OVX. The analysis revealed that the dendritic length at the level of the third order decreased in the immediately-OVX rats with E2 administration compared to corresponding vehicle group $\left({ }^{\mathrm{b}} P<0.05\right)$. (E) Length of branch order of the 10 -w-OVX rats with E2. The analysis of length per branch order of medium spiny NAcc neurons from 10-w-OVX rats with E2 did not show any difference.

related with its degree of connectivity and afferent activity [32]. For example, loss of principal afferents to spiny neurons during early development produces alterations in dendritic spines [33-38], while the decreased afferent activity by in vivo NMDA receptors blockade, reduces dendritic growth and branching of cerebellar and tectal neurons [3941]. It is known that NAcc medium spiny neurons receive glutamatergic inputs from prefrontal cortex and hippocampus [42-44] and recent reports suggest that OVX produces a decrease in the dendritic spines density at the level of hippocampus and prefrontal cortex. Therefore, a decrease of the glutamatergic inputs to medium spiny neurons of the NAcc may results in a decrease in the number of spines [32] as observed in 10-week control group in comparison to 2-week control group.

Data regarding the influence of ovarian hormones on the structure of neurons are contradictory. Thus, OVX results in a decrease in the number of dendritic spines in hippocampus and prefrontal cortex [12]. Contrarily, proteins MAP and Tau, involved in the development of dendrites and axons respectively, do not change in frontal cortex as a consequence of E2 or P treatment [45], or due to hormonal variations along the estrous cycle [46]. Similarly, density of dendritic spines in the hippocampal CA1 stratum radiatum, seems to be not related with the high levels of estrogens reached during proestrous [47]. These data support the idea that estrogenic actions on dendritic architecture is regulated by several factors including the time elapsed after OVX and the connectivity and afferent activity of some particular brain region. In this sense, removal of ovaries can increase the dendritic branch number and spine density of pyramidal cells from parietal cortex observed four months after an OVX [48], while an estrogenic treatment similar to used in the current study, decreases the number of branches of dendrites of layer II/III pyramidal cells in the entorhinal cortex [49]).

As mentioned, long-term removal of ovaries could be useful to study some characteristics of human postmenopause. Thus, the absence of an effect of E2 therapy in 10 -week rats seems to be comparable to some clinical data showing no benefit and even potential hazards of E2 treatment [50-52]. This idea has been supported by the observation that similar hormone levels act differently on the brain of a younger individual compared with an older one $[10,53,54]$ and by the fact that E2 applied 30 days, but no 10 days, after OVX does not produce any effect on cell density in the compact zone of the substantia nigra [55]. Similarly, E2 increases dendritic spine density in CA1 pyramidal cells of young female rats but such an effect is attenuated in aged monkeys [56], highlighting the role of the estrogenic milieu on synaptogenesis and supporting the hypothesis that long-term OVX rats could be a useful model to study some disorders associated to human menopause.

\section{CONCLUSION}

This study shows that both OVX and estrogenic replacement short- or long-term after this surgery can produce different actions on neuronal architecture in NAcc of rats. Due to the consequences of these changes, time frame after cessation of ovarian activity in women must be taken into account when beginning a hormone replacement therapy.

\section{ACKNOWLEDGEMENTS}

This work was supported by CONACyT Mexico (No. 57336) to GF, COFAA and SIP-IPN to OP. ARQ is recipient 
of a studentship from Escuela Superior de Medicina, IPN. GF and OP are members of the Researcher National System of Mexico. We thank Mr. Alan Larsen for checking the English-language.

\section{REFERENCES}

[1] Halbreich U, Kahn LS. Role of estrogen in the aetiology and treatment of mood disorders. CNS Drugs 2001; 15: 797-817.

[2] Li H, Satinoff E. Body temperature and sleep in intact and ovariectomized female rats. Am J Physiol 1996; 271: R1753-8.

[3] Osterlund MK, Witt MR, Gustafsson JA. Estrogen action in mood and neurodegenerative disorders: estrogenic compounds with selective properties-the next generation of therapeutics. Endocrine 2005; 8: 235-42.

[4] Picazo O, Estrada-Camarena E, Hernandez-Aragon A. Influence of the post-ovariectomy time frame on the experimental anxiety and the behavioural actions of some anxiolytic agents. Eur J Pharmacol 2006; 530: 88-94.

[5] Stahl SM. Estrogen makes the brain a sex organ. J Clin Psychiatry 1997; 58: 421-2.

[6] Adams MM, Fink SE, Shah RA, et al. Estrogen and aging affect the subcellular distribution of estrogen receptor-alpha in the hippocampus of female rats. J Neurosci 2002; 22: 3608-14

[7] Capriotti $T$. Inadequate cardiovascular disease prevention in women with physical disabilities. Rehabil Nurs 2006; 31: 94-101.

[8] Landry M, Di Paolo T. Effect of chronic estradiol, tamoxifen or raloxifene treatment on serotonin 5-HT1A receptor. Brain Res Mol Brain Res 2003; 112: 82-9.

[9] Raap DK, DonCarlos LL, Garcia F, et al. Ovariectomy-induced increases in hypothalamic serotonin-1A receptor function in rats are prevented by estradiol. Neuroendocrinology 2002; 76: 348-56.

[10] Adams MM, Shah RA, Janssen WG, Morrison JH. Different modes of hippocampal plasticity in response to estrogen in young and aged female rats. Proc Natl Acad Sci USA 2001; 98: 8071-6.

[11] Wallace $M$, Frankfurt $M$, Arellanos A, Inagaki T, Luine V. Impaired recognition memory and decreased prefrontal cortex spine density in aged female rats. Ann N Y Acad Sci 2007; 1097: 54-7.

[12] Wallace M, Luine V, Arellanos A, Frankfurt M. Ovariectomized rats show decreased recognition memory and spine density in the hippocampus and prefrontal cortex. Brain Res 2006; 1126: 176-82.

[13] De Castilhos J, Forti CD, Achaval M, Rasia-Filho AA. Dendritic spine density of posterodorsal medial amygdala neurons can be affected by gonadectomy and sex steroid manipulations in adult rats: a Golgi study. Brain Res 2008; 1240: 73-81.

[14] Leranth C, Roth RH, Elsworth JD, Naftolin F, Horvath TL, Redmond DE, Jr. Estrogen is essential for maintaining nigrostriatal dopamine neurons in primates: implications for Parkinson's disease and memory. J Neurosci 2000; 20: 8604-9.

[15] Tang Y, Janssen WG, Hao J, et al. Estrogen replacement increases spinophilin-immunoreactive spine number in the prefrontal cortex of female rhesus monkeys. Cereb Cortex 2004; 14: 215-23.

[16] Leranth C, Hajszan T, MacLusky NJ. Androgens increase spine synapse density in the CA1 hippocampal subfield of ovariectomized female rats. J Neurosci 2004; 24: 495-9.

[17] MacLusky NJ, Luine VN, Hajszan T, Leranth C. The 17alpha and 17 beta isomers of estradiol both induce rapid spine synapse formation in the CA1 hippocampal subfield of ovariectomized female rats. Endocrinology 2005; 146: 287-93.

[18] Gurkan L, Ekeland A, Gautvik KM, Langeland N, Ronningen H, Solheim LF. Bone changes after castration in rats: a model for osteoporosis. Acta Orthop Scand 1986; 57: 67-70.

[19] Kalu DN, Liu CC, Hardin RR, Hollis BW. The aged rat model of ovarian hormone deficiency bone loss. Endocrinology 1989; 124: 7-16.

[20] Kalu DN. The ovariectomized rat model of postmenopausal bone loss. Bone Miner 1991; 15: 175-91.

[21] Lam KK, Hu CT, Ou TY, Yen MH, Chen HI. Effects of oestrogen replacement on steady and pulsatile haemodynamics in ovariectomized rats. Br J Pharmacol 2002; 136: 811-8.

[22] Flores G, Alquicer G, Silva-Gomez AB, et al. Alterations in dendritic morphology of prefrontal cortical and nucleus accumbens neurons in post-pubertal rats after neonatal excitotoxic lesions of the ventral hippocampus. Neuroscience 2005; 133: 463-70.
[23] Gibb R, Kolb B. A method for vibratome sectioning of Golgi-Cox stained whole rat brain. J Neurosci Methods 1998; 79: 1-4.

[24] Juarez I, Gratton A, Flores G. Ontogeny of altered dendritic morphology in the rat prefrontal cortex, hippocampus and nucleus accumbens following Caesarean delivery and birth anoxia. J Comp Neurol 2008; 507: 1734-47.

[25] Paxinos G, Watson C. The rat brain in stereotaxic coordinates. $2^{\text {nd }}$ ed. New York: Academic Press 1986.

[26] Kolb B, Forgie M, Gibb R, Gorny G, Rowntree S. Age, experience and the changing brain. Neurosci Biobehav Rev 1998; 22: 143-59.

[27] Robinson TE, Kolb B. Persistent structural modifications in nucleus accumbens and prefrontal cortex neurons produced by previous experience with amphetamine. J Neurosci 1997; 17: 84917.

[28] Martinez-Tellez R, Gomez-Villalobos MJ, Flores G. Alteration in dendritic morphology of cortical neurons in rats with diabetes mellitus induced by streptozotocin. Brain Res 2005; 1048: 108-15.

[29] Silva-Gomez AB, Bermudez M, Quirion R, Srivastava LK, Picazo O, Flores G. Comparative behavioral changes between male and female postpubertal rats following neonatal excitotoxic lesions of the ventral hippocampus. Brain Res 2003; 973: 285-92.

[30] Vega E, Gomez-Villalobos MJ, Flores G. Alteration in dendritic morphology of pyramidal neurons from the prefrontal cortex of rats with renovascular hypertension. Brain Res 2004; 1021: 112-8.

[31] Sholl DA. Dendritic organization in the neurons of the visual and motor cortices on the cat. J Anat 1953; 87: 387-406.

[32] McAllister AK. Cellular and molecular mechanisms of dendrite growth. Cereb Cortex 2000; 10: 963-73.

[33] Friedlander MJ, Stanford LR, Sherman SM. Effects of monocular deprivation on the structure-function relationship of individual neurons in the cat's lateral geniculate nucleus. J Neurosci 1982; 2: 321-30.

[34] Globus A, Scheibel AB. Synaptic loci on parietal cortical neurons: terminations of the corpus callosum fibers. Science 1967; 156: 1127-9.

[35] Guillery RW. The effect of lid suture upon the growth of cells in the dorsal lateral geniculate nucleus of kittens. J Comp Neurol 1973; 148: 417-22.

[36] Heumann D, Rabinowicz T. Postnatal development of the visual cortex of the mouse after enucleation at birth. Exp Brain Res 1982; 46: 99-106.

[37] McMullen NT, Glaser EM. Auditory cortical responses to neonatal deafening: pyramidal neuron spine loss without changes in growth or orientation. Exp Brain Res 1988; 72: 195-200.

[38] Wiesel TN, Hubel DH. Effects of visual deprivation on morphology and physiology of cells in the cats lateral geniculate body. J Neurophysiol 1963; 26: 978-93.

[39] Kalb RG. Regulation of motor neuron dendrite growth by NMDA receptor activation. Development 1994; 120: 3063-71.

[40] Rajan I, Witte S, Cline HT. NMDA receptor activity stabilizes presynaptic retinotectal axons and postsynaptic optic tectal cell dendrites in vivo. J Neurobiol 1999; 38: 357-68.

[41] Vogel MW, Prittie J. Purkinje cell dendritic arbors in chick embryos following chronic treatment with an N-methyl-D-aspartate receptor antagonist. J Neurobiol 1995; 26: 537-52.

[42] Aylward RL, Totterdell S. Neurons in the ventral subiculum, amygdala and entorhinal cortex which project to the nucleus accumbens: their input from somatostatin-immunoreactive boutons. J Chem Neuroanat 1993; 6: 31-42.

[43] Jay TM, Witter MP. Distribution of hippocampal CA1 and subicular efferents in the prefrontal cortex of the rat studied by means of anterograde transport of phaseolus vulgarisleucoagglutinin. J Comp Neurol 1991; 313: 574-86.

[44] Thierry AM, Gioanni Y, Degenetais E, Glowinski J. Hippocampoprefrontal cortex pathway: anatomical and electrophysiological characteristics. Hippocampus 2000; 10: 411-9.

[45] Reyna-Neyra A, Camacho-Arroyo I, Ferrera P, Arias C. Estradiol and progesterone modify microtubule associated protein 2 content in the rat hippocampus. Brain Res Bull 2002; 58: 607-12.

[46] Reyna-Neyra A, Arias C, Ferrera P, Morimoto S, Camacho-Arroyo I. Changes in the content and distribution of microtubule associated protein 2 in the hippocampus of the rat during the estrous cycle. J Neurobiol 2004; 60: 473-80.

[47] Brusco J, Wittmann R, de Azevedo MS, et al. Plasma hormonal profiles and dendritic spine density and morphology in the hippocampal CA1 stratum radiatum, evidenced by light 
microscopy, of virgin and postpartum female rats. Neurosci Lett 2008; 438: 346-50.

[48] Stewart J, Kolb B. Dendritic branching in cortical pyramidal cells in response to ovariectomy in adult female rats: suppression by neonatal exposure to testosterone. Brain Res 1994; 654: 149-54.

[49] Flores C, Salmaso N, Cain S, Rodaros D, Stewart J. Ovariectomy of adult rats leads to increased expression of astrocytic basic fibroblast growth factor in the ventral tegmental area and in dopaminergic projection regions of the entorhinal and prefrontal cortex. J Neurosci 1999; 19: 8665-73.

[50] Genazzani AR, Pluchino N, Luisi S, Luisi M. Estrogen, cognition and female ageing. Human Reprod Update 2007; 13: 175-87.

[51] Morrison JH, Brinton RD, Schmidt PJ, Gore AC. Estrogen, menopause, and the aging brain: how basic neuroscience can inform hormone therapy in women? J Neurosci 2006; 26: 1033248
[52]

aged rats. Neurobiol Aging 2000; 21. 107-16. replacement therapy on cholinergic basal forebrain neurons and cortical cholinergic innervation in young and aged ovariectomized rhesus monkeys. J Comp Neurol 2004; 472: 193-207.

[55] Leranth C, Shanabrough M, Redmond DE. Gonadal hormones are responsible for maintaining the integrity of spine synapses in the CA1 hippocampal subfield of female nonhuman primates. J Comp Neurol 2002; 447: 34-42.

[56] Hao J, Janssen WG, Tang Y, et al. Estrogen increases the number of spinophilin-immunoreactive spines in the hippocampus of young and aged female rhesus monkeys. J Comp Neurol 2003; 465: 54050

Received: April 14, 2009

Revised: September 3, 2009

Accepted: September 4, 2009

(C) Quiñones et al.; Licensee Bentham Open.

This is an open access article licensed under the terms of the Creative Commons Attribution Non-Commercial License (http://creativecommons.org/licenses/by$\mathrm{nc} / 3.0 /$ ) which permits unrestricted, non-commercial use, distribution and reproduction in any medium, provided the work is properly cited. 